\title{
Consumo de tabaco en México. Resultados de las Encuestas Nacionales de Salud 2000 y 2006
}

\author{
Aremis Villalobos, Act, MD, (I) Rosalba Rojas, MC, PhD. ${ }^{(1)}$
}

\section{Villalobos A, Rojas R. Consumo de tabaco en México. Resultados de las Encuestas Nacionales de Salud 2000 y 2006. Salud Publica Mex 2007;49 supl 2:SI47-SI54.}

\section{Resumen}

Objetivo. Analizar el consumo de tabaco en adolescentes y adultos a partir de la ENSA 2000 y la ENSANUT 2006. Material y métodos. Se presenta información del consumo de tabaco en todo México proveniente de los cuestionarios aplicados a adolescentes y adultos. El análisis estadístico consistió en obtener prevalencias e intervalos de confianza al 95\%. Resultados. En el 2000, 9.7\% (IC95\%: 8.9 a I0.6) de los adolescentes declaró que había fumado por lo menos cien cigarrillos de tabaco durante toda su vida, pero en el 2006 se encontró que fue 7.6\% (IC95\%:7.0 a 8.2). Las prevalencias en los adultos en 2000 y 2006 fueron $12.9 \%$ (IC95\%: I I.8 a I4. I) y 13.3\% (IC95\%: 12.8 a I3.8) en fumadores diarios y $9.4 \%$ (IC95\%: 8.9 a 9.9 ) y $5.6 \%$ (IC95\%: 5.3 a 6.0 ) en fumadores ocasionales. Conclusión. Los resultados en este estudio muestran una reducción significativa en el consumo de tabaco en fumadores ocasionales; sin embargo, es necesario continuar con estudios específicos en el tema utilizando definiciones que se emplean en el contexto internacional.

Palabras clave: prevalencias de consumo de tabaco; ENSA 2000; ENSANUT 2006; México
Villalobos A, Rojas R.

Tobacco smoke in Mexico. Results from the National

Health Surveys 2000 and 2006.

Salud Publica Mex 2007;49 suppl 2:SI47-SI 54.

\section{Abstract}

Objective. To analyze tobacco consumption in teenagers and adults based on the National Health Surveys 2000 and 2006. Material and Methods. Tobacco consumption information is presented at national level, from questionnaires answered by teenagers and adults. Statistical analysis consisted in general and specific prevalences and intervals obtained at $95 \%$ confidence. Results. 9.7\% (95\% Cl: 8.9-10.6) teenagers stated that they have smoked at least 100 cigarettes during their life, in 2000 , and $7.6 \%$ (95\% Cl: 7.0-8.2) in 2006. Prevalences in adults in 2000 and 2006 were: I $2.9 \%$ (95\% Cl: II.8 a |4.I) and I3.3\% (95\% Cl: 12.8 a I3.8) in daily smokers and $9.4 \%$ (95\% Cl: 8.9 a 9.9$)$ and $5.6 \%$ (95\% Cl: 5.3 a 6.0) in occasionally smokers. Conclusion. Results from this study show a decrement in tobacco consumption in occasionally smokers. Nevertheless, keep doing specific studies about this issue, including the use of international definitions, is necessary.

Key words: tobacco smoke prevalence; ENSA 2000; ENSANUT 2006; Mexico

(I) Instituto Nacional de Salud Pública. México.

Fecha de recibido: 4 de abril de 2007 • Fecha de aprobado: 30 de abril de 2007 Solicitud de sobretiros: Dra. Rosalba Rojas, Instituto Nacional de Salud Pública,Avenida Universidad 655, Col. Santa María Ahuacatitlán, 62508, Cuernavaca, Morelos, México. Correo electrónico: rrojas@insp.mx 
$\mathrm{E}$ consumo de tabaco es una de las principales causas de muertes evitables y de discapacidad en el mundo y constituye uno de los factores de riesgo de enfermedad, discapacidad y muerte más importantes de México, pa s en donde se relaciona con al menos 40 causas de muerte, dentro de las que destacan la enfermedad pulmonar obstructiva crónica, el enfisema pulmonar, el cáncer de pulmón y de laringe y enfermedades del corazón y cerebrovasculares. Se estima que los fumadores mueren de manera prematura, y pierden en promedio de 20 a 25 años de vida productiva. ${ }^{1}$

En el Programa de Acción de adicciones se menciona que el Banco Mundial señala al tabaco como un problema mayor en las poblaciones pobres, jóvenes, de bajo nivel educativo y bajos ingresos. Las muertes atribuibles al consumo de tabaco representan sólo $5 \%$ en poblaciones con educación media y superior, mientras el porcentaje se incrementa a 19\% en los grupos que tienen primaria como máximo grado académico. Además, se reconoce al grupo de adolescentes como grupo de alto riesgo ya que más de $60 \%$ de los fumadores se inicia desde los 13 años y más de $90 \%$ antes de los 20 años. $^{2}$

Según los datos de las cl nicas de tabaco del Sistema de Vigilancia Epidemiológica de las Adicciones (SISVEA), en el 2003 55\% de los individuos que acudieron a dichas cl nicas comenzó el hábito entre los 10 y los 14 años de edad; para el año 2004, 80\% inició el consumo de tabaco antes de los 18 años. ${ }^{3}$

En México, en los últimos años se realizaron varias encuestas que proporcionan información sobre el consumo de tabaco en diferentes poblaciones, entre ellas la Encuesta sobre Tabaquismo en Jóvenes México 2003 (ETJ), que se aplicó en 10 ciudades, y que señala que $19.9 \%$ de los estudiantes son fumadores actuales, $19.6 \%$ en hombres y $19.1 \%$ en mujeres. ${ }^{4}$ Los datos sobre el consumo de tabaco que se obtuvieron en las Encuestas Nacionales de Adicciones (ENA) durante el periodo 1988 a 1998, realizadas en población de 12 a 65 años en áreas urbanas, muestran un discreto incremento en la prevalencia de fumadores. En 1988, la prevalencia fue de $25.8 \%,{ }^{5}$ mientras que en 1993 fue de $25.1 \%$, ${ }^{6}$ y en 1998 de $27.7 \% .^{7}$ Los resultados de estas tres encuestas muestran que la población fumadora en México creció a expensas del grupo de jóvenes, es decir que en México no se observó una clara tendencia al crecimiento entre la población fumadora, sino que el efecto del crecimiento se observó en algunos grupos espec ficos como el de las mujeres jóvenes y la edad de inicio en el consumo de tabaco se acercó cada vez más a la de los hombres. Por su parte, en el grupo de adultos, los resultados de dichas encuestas sugieren una estabilización de la epidemia, ya que no se observan variaciones claras ni para el grupo de hombres ni para el de mujeres. ${ }^{8}$
La Encuesta Nacional de Adicciones del año 2002 hizo evidente que $23.5 \%$ de la población nacional de 12 a 65 años fuma. Al analizar a la población del mismo grupo de edad por tamaño de localidad, los resultados indican que $26.4 \%$ de los que residen en áreas urbanas y $14.3 \%$ de la población residente en áreas rurales son fumadores activos. ${ }^{9}$

La Encuesta Nacional de Salud y Nutrición 2006 (ENSANUT 2006) es la encuesta más compleja que se haya realizado dentro del sistema de Encuestas Nacionales de Salud en México; recaba información actualizada del estado de salud y nutrición de la población mexicana, permite obtener prevalencias de padecimientos y en algunos casos permite realizar comparaciones con resultados obtenidos en otras encuestas. ${ }^{10} \mathrm{El}$ protocolo de la ENSANUT 2006 contó con la aprobación del comité de ética del Instituto Nacional de Salud Pública. Durante el levantamiento de dicha encuesta, se obtuvo el consentimiento informado de los participantes.

El objetivo de este trabajo consiste en analizar la prevalencia de consumo de tabaco a partir de la Encuesta Nacional de Salud (ENSA 2000) y la fuente de información más actualizada que se tiene, es decir, la ENSANUT 2006, con el fin de poner a disposición información actualizada sobre los indicadores del consumo de tabaco en jóvenes y adultos de México.

\section{Material y métodos}

En este trabajo se utilizaron las bases de datos de la ENSA 2000 y la ENSANUT 2006. Las dos encuestas se diseñaron para recabar información relacionada con el estado de salud de la población mexicana. Los diseños muestrales de las dos encuestas fueron probabil sticos, polietápicos, estratificados y por conglomerados. Ambas encuestas generan información representativa en tamaño de localidad por entidad federativa y del conjunto nacional.

Las estimaciones se presentan como prevalencias puntuales y sus intervalos de confianza al 95\%. Se calcularon prevalencias generales y espec ficas de consumo de tabaco para algunos subgrupos de interés. El procesamiento estad stico de la información tomó en cuenta el diseño muestral complejo de las dos encuestas y se realizó con el paquete estad stico SPSS versión 13.0.

Los resultados que se presentan provienen en primer lugar de los cuestionarios de adolescentes y de adultos, y de ellos el primero proporciona información referida a la población de 10 a 19 años de edad, mientras que el segundo provee información sobre la población con 20 años o más. En ambos cuestionarios aplicados se pregunta acerca del consumo de tabaco y otros factores de riesgo. 
Las principales definiciones que se emplean en este trabajo son: fumador actual, que incluye fumador diario y fumador ocasional, ex fumador y no fumador, en el caso de la población adulta. No fue posible establecer una definición más precisa de fumador adolescente debido a que no se contó con información sobre el consumo de tabaco durante los últimos $30 \mathrm{~d}$ as. A dicha población se le preguntó si hab a fumado por lo menos 100 cigarrillos de tabaco durante toda su vida, número de cigarrillos y frecuencia de consumo. En el caso de los adultos se consideró como fumador actual cuando declaró que hab a fumado por lo menos 100 cigarrillos de tabaco durante toda su vida y que en la actualidad fumaba; fumador diario se refiere a aquel que lo realiza a diario y cuando la frecuencia de consumo fue en un intervalo mayor se consideró que se trataba de un fumador ocasional. Se definió como ex fumador a aquella persona que declaró que hab a fumado por lo menos 100 cigarrillos de tabaco durante toda su vida y que en el presente no fumaba. Los no fumadores fueron los que declararon haber fumado menos de 100 cigarrillos en su vida o que nunca hab an fumado.

En el análisis de consumo de bebidas alcohólicas por parte de adolescentes se consideró como bebedor al joven que reconoció haber consumido alcohol aunque fuera en ocasiones; no resultó posible establecer una definición más precisa de bebedor regular debido a que no se contó con el mismo tipo de información detallada sobre el consumo de bebidas alcohólicas, ya que en el año 2000 se pregunta acerca del consumo de una copa o vaso completo de alguna bebida alcohólica, mientras que en el 2006 se pregunta acerca del consumo de cinco copas o más en alguna ocasión. En el caso de población adulta en ambos años, se utilizó la pregunta de consumo actual de bebidas alcohólicas.

Al analizar la variable de tamaño de la localidad, en la ENSA 2000 se estratificó en localidades con un número igual o mayor a 15000 habitantes y localidades rurales fueron aquellas con un número menor de habitantes (localidades entre 1 y 14999 habitantes). En el caso de la ENSANUT 2006, se consideraron estratos por tamaño de localidad en tres grupos: ciudad o área metropolitana, complemento urbano y rural. Las ciudades y áreas metropolitanas incluyen ciudades, áreas metropolitanas y el resto de las ciudades de 100000 y más habitantes y/ o capitales de estado; el complemento urbano incluyó a todas las localidades de 2500 a 99999 habitantes y en las localidades rurales se incluyeron aquellas con menos de 2500 habitantes. Para hacer comparables los tamaños de localidad, se utilizaron las bases de datos existentes con los tamaños de las localidades en el pa s del Instituto Nacional de Estad stica Geograf a e Informática (INEGI) según el XII Censo General de
Población y Vivienda 2000, los cuales se unieron a la base de datos de la ENSA 2000. As, el análisis en este estudio se presenta para localidades con menos de 2500 habitantes y localidades con 2500 o más habitantes.

\section{Resultados}

\section{Características de consumo de tabaco en adolescentes}

Al analizar las preguntas de consumo de tabaco se encontró que, en el año 2000, 9.7\% (IC95\%: 8.9 a 10.6) de los adolescentes hab a fumado por lo menos 100 cigarrillos de tabaco durante toda su vida y que en 2006 la proporción de adolescentes en el mismo rango de edad fue de 7.6\% (IC95\%: 7.0 a 8.2). Al estratificar por género se encontraron diferencias significativas en ambas encuestas. La prevalencia de consumo de tabaco en el año 2000 fue de 14.5\% (IC95\%: 13.1 a 16.0) en hombres, pero en 2006 la prevalencia disminuyó de manera significativa a 10.8\% (IC95\%: 9.9 a 11.8). En el caso de las mujeres fue de $5.0 \%$ (IC95\%: 4.4 a 5.8) en 2000 y $4.3 \%$ (IC95\%: 3.7 a 4.8) en 2006 (cuadro I).

De manera adicional, las prevalencias de consumo de más de 100 cigarrillos durante la vida del adolescente se incrementan con la edad, con la siguiente progresión: en la población entre 10 y 12 años fue de $1.5 \%$ (IC95\%: 1.0 a 2.3) y de $0.4 \%$ (IC95\%: 0.2 a 0.8 ) en 2000 y el 2006, respectivamente. En el grupo de 13 a 15 años, la prevalencia fue de $5.4 \%$ (IC95\%: 4.6 a 6.3) en 2000 y disminuyó significativamente a 3.5\% (IC95\%: 2.9 a 4.3) en 2006. Para finalizar, entre los adolescentes de 16 a 19 años no se encontró diferencia significativa en las prevalencias de 2000 y 2006, ya que fueron de $20.5 \%$ (IC95\%: 18.7 a 22.5) y 17.6\% (IC95\%: 16.3 a 19.0), respectivamente (cuadro II).

Para el grupo que declaró consumir bebidas alcohólicas la prevalencia fue de $28.3 \%$ (IC95\%: 25.8 a 31.0) en 2000 y de $38.0 \%$ (IC95\%: 35.2 a 40.8) en 2006, que fueron prevalencias significativamente diferentes.

Los datos muestran diferencias significativas por tamaño de localidad. En las localidades con menos de 2500 habitantes la prevalencia de consumo de tabaco fue de $5.6 \%$ (IC95\%: 4.6 a 6.7) en 2000 y de $3.7 \%$ (IC95\%: 3.1 a 4.4) en 2006. En localidades con 2500 habitantes o más, las prevalencias estimadas son de $11.4 \%$ (IC95\%: 10.3 a 12.6) y de $9.1 \%$ (IC95\%: 8.3 a 9.9), respectivamente.

También se observaron diferencias significativas entre las prevalencias de consumo de tabaco en la población que declaró asistir a la escuela en el momento del levantamiento de la encuesta. En 2000, la prevalencia fue de $6.4 \%$ (IC95\%: 5.7 a 7.2) y en 2006 de 4.7\% (IC95\%: 4.1 a 5.3$)$. 


\section{Cuadro I}

Distribución PORCENTUAL de ADOLESCENTES de 10 A 19 AÑOS DE EDAD SEGÚN CONSUMO de POR LO MENOS 100 CIGARRILLOS A LO LARGO DE SU VIDA Y POR GÉNERO, 2000-2006

\begin{tabular}{|c|c|c|c|c|c|c|}
\hline & & ENSA 2000 & & & NSANUT 20 & \\
\hline & $\begin{array}{c}\text { Hombres } \\
\text { porcentaje } \\
\text { (IC95\%) }\end{array}$ & $\begin{array}{c}\text { Mujeres } \\
\text { porcentaje } \\
\text { (IC95\%) }\end{array}$ & $\begin{array}{c}\text { Total } \\
\text { porcentaje } \\
\text { (IC95\%) }\end{array}$ & $\begin{array}{c}\text { Hombres } \\
\text { porcentaje } \\
\text { (IC95\%) }\end{array}$ & $\begin{array}{c}\text { Mujeres } \\
\text { porcentaje } \\
\text { (IC95\%) }\end{array}$ & $\begin{array}{c}\text { Total } \\
\text { porcentaje } \\
\text { (IC95\%) }\end{array}$ \\
\hline $\begin{array}{l}\text { ¿Has fum } \\
\text { de tabac }\end{array}$ & & & & & & \\
\hline Sí & $\begin{array}{c}14.5 \\
(13.1 \text { a } 16.0)\end{array}$ & $\begin{array}{c}5.0 \\
(4.4 \text { a } 5.8)\end{array}$ & $\begin{array}{c}9.7 \\
(8.9 \text { a } 10.6)\end{array}$ & $\begin{array}{c}10.8 \\
(9.9 \text { a II.8) }\end{array}$ & $\begin{array}{c}4.3 \\
(3.7 \text { a } 4.8)\end{array}$ & $\begin{array}{c}7.6 \\
(7.0 \text { a } 8.2)\end{array}$ \\
\hline $\mathrm{No}^{\ddagger}$ & $\begin{array}{c}85.5 \\
(84.0 \text { a } 86.9)\end{array}$ & $\begin{array}{c}95.0 \\
(94.2 \text { a } 95.6)\end{array}$ & $\begin{array}{c}90.3 \\
(89.4 \text { a } 91.1)\end{array}$ & $\begin{array}{c}89.2 \\
(88.2 \text { a } 90.1)\end{array}$ & $\begin{array}{c}95.7 \\
(95.2 \text { a } 96.3)\end{array}$ & $\begin{array}{c}92.4 \\
(91.8 \text { a } 93.0)\end{array}$ \\
\hline * $4.8 \% d$ & & & & & & \\
\hline ‡ Incluye & han fumado & & & & & \\
\hline
\end{tabular}

Cuadro II

Prevalencias de consumo de 100 o más cigarrillos en adolescentes durante la vida de la persona, 2000-2006

\begin{tabular}{|c|c|c|c|c|}
\hline & & & ENSA & 2006 \\
\hline & $\begin{array}{l}\text { Consu } \\
\text { cigarri }\end{array}$ & $\begin{array}{l}0 \text { o más } \\
\text { e la vida }\end{array}$ & $\begin{array}{l}\text { Consum } \\
\text { cigarrillo }\end{array}$ & $\begin{array}{l}0 \text { o más } \\
\text { te la vida }\end{array}$ \\
\hline & $\begin{array}{c}\text { Sí } \\
\text { Porcentaje (IC95\%) }\end{array}$ & $\begin{array}{c}\mathrm{No}^{*} \\
\text { Porcentaje (IC95\%) }\end{array}$ & $\begin{array}{c}\text { Sí } \\
\text { Porcentaje (IC95\%) }\end{array}$ & $\begin{array}{c}\mathrm{No}^{*} \\
\text { Porcentaje (IC95\%) }\end{array}$ \\
\hline Grupos de edad & & & & \\
\hline 10 a 12 años & 1.5 & 98.5 & 0.4 & 99.6 \\
\hline & $(1.0$ a 2.3$)$ & (97.7 a 99.0$)$ & $(0.2$ a 0.8$)$ & (99.2 a 99.8) \\
\hline 13 a 15 años & 5.4 & 94.6 & 3.5 & 96.5 \\
\hline & $(4.6$ a 6.3$)$ & (93.7 a 95.4) & $(2.9$ a 4.3$)$ & $(95.7$ a 97.1$)$ \\
\hline 16 a 19 años & 20.5 & 79.5 & 17.6 & 82.4 \\
\hline & (18.7 a 22.5$)$ & (77.5 a 8I.3) & $(16.3$ a 19.0$)$ & $(81.0$ a 83.7$)$ \\
\hline Consumo de alcohol & & & & \\
\hline Sí & 28.3 & 71.7 & 38.0 & 62.0 \\
\hline & $(25.8$ a 31.0$)$ & $(69.0$ a 74.2$)$ & (35.2 a 40.8$)$ & (59.2 a 94.8) \\
\hline No & 3.1 & 96.9 & 3.9 & 96.1 \\
\hline & $(2.6$ a 3.7$)$ & $(96.3$ a 97.4$)$ & (3.4 a 4.4) & $(95.6$ a 96.6$)$ \\
\hline Tamaño de localidad & & & & \\
\hline menos de 2500 habitantes & 5.6 & 94.4 & 3.7 & 96.3 \\
\hline & $(4.6$ a 6.7$)$ & $(93.3$ a 95.4$)$ & (3.I a 4.4$)$ & $(95.6$ a 96.9$)$ \\
\hline 2500 habitantes o más & $1 \mathrm{I} .4$ & 88.6 & 9.1 & 90.9 \\
\hline & $(10.3$ a I2.6) & (87.4 a 89.7) & $(8.3$ a 9.9$)$ & (90.1 a 91.7) \\
\hline Asistencia actual a la escuela & & & & \\
\hline Sí & 6.4 & 93.6 & 4.7 & 95.3 \\
\hline & $(5.7$ a 7.2$)$ & (92.8 a 94.3$)$ & (4.I a 5.3$)$ & (94.7 a 95.9) \\
\hline No & 19.0 & 81.0 & 17.2 & 82.8 \\
\hline & $(\mid 7.0$ a 21.1$)$ & $(78.9$ a 83.0$)$ & (I5.6 a I8.8) & $(81.2$ a 84.4$)$ \\
\hline Total & 9.7 & 90.3 & 7.6 & 92.4 \\
\hline & $(8.9$ a 10.6$)$ & (89.4 a 9I.I) & (7.0 a 8.2) & (91.8 a 93.0) \\
\hline $\mathrm{N}^{\ddagger}$ & 2047794 & 19043767 & 735934 & 21139036 \\
\hline${ }^{*}$ Incluye a los que no fuman y & umido menos de & & & \\
\hline ‡ Incluye sólo casos válidos & & & & \\
\hline
\end{tabular}




\section{Características de consumo de tabaco en adultos}

Al analizar la información de los adultos en el consumo $\mathrm{m}$ nimo de 100 cigarrillos, si fuma en el presente y la frecuencia de consumo, se encontró que en al caso de los fumadores diarios fue de $12.9 \%$ (IC95\%: 11.8 a 14.1) en el año 2000 y $13.3 \%$ (IC95\%: 12.8 a 13.8) en el 2006. Se encontraron diferencias significativas en los casos del fumador ocasional, con $9.4 \%$ (IC95\%: 8.9 a 9.9) y $5.6 \%$ (IC95\%: 5.3 a 6.0) en 2000 y 2006, respectivamente. En el caso de ex fumadores, se encontraron diferencias significativas entre los hombres, con 21.6\% (IC95\%: 20.5 a 22.7) en 2000 y 16.5\% (IC95\%: 15.6 a 17.4) en 2006 (cuadro III).

Además, se encontraron diferencias significativas en las proporciones de fumador ocasional entre los distintos grupos de edad, con las mayores diferencias entre 2000 y 2006 en el grupo de 40 a 49 años con $9.2 \%$ (IC95\%: 8.2 a 10.4) y $5.3 \%$ (IC95\%: 4.6 a 6.1). Se observaron mayores prevalencias en las localidades con 2500 habitantes o más con $9.8 \%$ (IC95\%: 9.2 a 10.4), cifra que disminuyó a 6.0\% (IC95\%: 5.6 a 6.4) en el 2006. En los que consumen alcohol en el presente, se encontró que la prevalencia en fumadores ocasionales disminuyó de manera significativa en los dos años analizados al pasar de $17.4 \%$ (IC95\%: 16.4 a 18.5) en 2000 a $12.0 \%$ (IC95\%: 11.1 a 12.9) en el 2006 (cuadro IV).
En las prevalencias de los ex fumadores se encontraron diferencias significativas en los diferentes grupos de edad, con las cifras mayores en el grupo de 70 a 79 años en donde alcanzó 26.8\% (IC95\%: 23.6 a 30.2) en 2000 y $19.5 \%$ (IC95\%: 17.3 a 22.0) en 2006. También se encontró que en las localidades con 2500 habitantes o más la prevalencia fue de 14.3\% (IC95\%: 13.5 a 15.1) en 2000 y disminuyó a $11.5 \%$ (IC95\%: 10.9 a 12.1) en 2006.

\section{Conclusión}

Los resultados en este estudio muestran una reducción significativa en el consumo de 100 o más cigarrillos en los adolescentes de 10 a 19 años. En el 2000, 9.7\% (IC95\%: 8.9 a 10.6) de los adolescentes entre 10 y 19 años de edad declaró que hab a fumado por lo menos 100 cigarrillos de tabaco durante toda su vida, un porcentaje que disminuyó a 7.6\% (IC95\%: 7.0 a 8.2) en 2006. En los adultos con 20 años o más, se observa una reducción significativa en los fumadores ocasionales, con $9.4 \%$ (IC $95 \%$ : 8.9 a 9.9) en el año 2000 y 5.6\% (IC95\%: 5.3 a 6.0) en el 2006.

Los datos analizados muestran que existen diferencias significativas en adolescentes que consumieron 100 cigarrillos o más entre las prevalencias de hombres, pero lamentablemente en las prevalencias de mujeres no se observó lo mismo. En el caso del análisis estratificado por grupos de edad, se encontraron diferencias significa-

\section{Cuadro III}

DistribuCIÓN PORCENTUAL DE LA POBLACIÓN DE 20 AÑOS Y MÁS EDAD QUE CONSUMIÓ POR LO MENOS I00 CIGARRILLOS DE TABACO DURANTE TODA LA VIDA Y POR GÉNERO

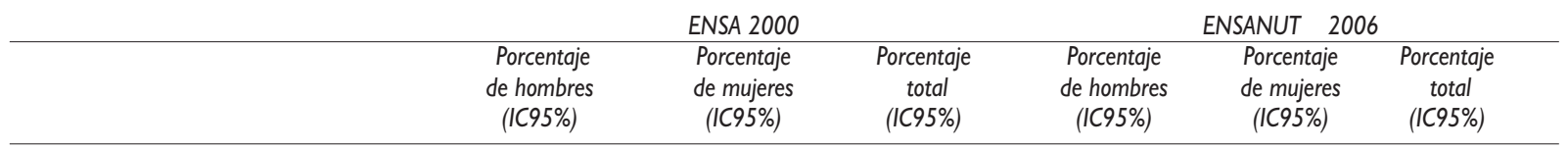

Has fumado por lo menos 100 cigarrillos de tabaco durante toda la vida

Sí

\begin{tabular}{|c|c|c|c|c|c|c|}
\hline fumador diario* & $\begin{array}{c}20.5 \\
(18.8 \text { a 22.3) }\end{array}$ & $\begin{array}{c}6.2 \\
(5.4 \text { a } 7.1)\end{array}$ & $\begin{array}{c}12.9 \\
(11.8 \text { a } \mid 4.1)\end{array}$ & $\begin{array}{c}21.6 \\
(20.6 \text { a } 22.5)\end{array}$ & $\begin{array}{c}6.5 \\
(6.0 \text { a } 7.0)\end{array}$ & $\begin{array}{c}13.3 \\
(12.8 \mathrm{a} \mathrm{I3.8)})\end{array}$ \\
\hline fumador ocasional $^{\ddagger}$ & $\begin{array}{c}15.3 \\
(14.3 \text { a } 16.4)\end{array}$ & $\begin{array}{c}4.0 \\
(3.6 \text { a } 4.5)\end{array}$ & $\begin{array}{c}9.4 \\
(8.9 \text { a 9.9) }\end{array}$ & $\begin{array}{c}8.8 \\
\text { (8.1 a 9.5) }\end{array}$ & $\begin{array}{c}3.0 \\
(2.6 \text { a 3.5) }\end{array}$ & $\begin{array}{c}5.6 \\
(5.3 \mathrm{a} 6.0)\end{array}$ \\
\hline ex fumador $\S$ & $\begin{array}{c}21.6 \\
(20.5 \text { a } 22.7)\end{array}$ & $\begin{array}{c}6.9 \\
(6.4 \text { a } 7.4)\end{array}$ & $\begin{array}{c}13.8 \\
(|3.2 \mathrm{a}| 4.5)\end{array}$ & $\begin{array}{c}16.5 \\
(15.6 \text { a I7.4) }\end{array}$ & $\begin{array}{c}6.0 \\
(5.5 \text { a 6.6) }\end{array}$ & $\begin{array}{c}10.8 \\
(10.2 \mathrm{a} \mid \mathrm{I} .3)\end{array}$ \\
\hline No & $\begin{array}{c}27.3 \\
(25.9 \text { a } 28.6)\end{array}$ & $\begin{array}{c}37.7 \\
(35.8 \text { a 39.6) }\end{array}$ & $\begin{array}{c}32.8 \\
(31.4 \text { a } 34.2)\end{array}$ & $\begin{array}{l}\text { I5.I } \\
(14.2 \text { a } 15.9)\end{array}$ & $\begin{array}{c}\text { II.I } \\
(10.4 \mathrm{a} \mid \mathrm{I} .8)\end{array}$ & $\begin{array}{c}12.9 \\
(12.3 \mathrm{a} \mathrm{I3.5})\end{array}$ \\
\hline Nunca ha fumado & $\begin{array}{c}15.3 \\
(13.9 \text { a } 16.8)\end{array}$ & $\begin{array}{c}45.1 \\
(43.1 \text { a } 47.2)\end{array}$ & $\begin{array}{c}3 \text { I.I } \\
(29.4 \text { a } 32.8)\end{array}$ & $\begin{array}{c}38.1 \\
(37.0 \text { a } 39.2)\end{array}$ & $\begin{array}{c}73.4 \\
(72.4 \text { a } 74.4)\end{array}$ & $\begin{array}{c}57.4 \\
(56.6 \text { a } 58.2)\end{array}$ \\
\hline
\end{tabular}

\footnotetext{
* Personas que fuman en la actualidad y la frecuencia es diaria

₹ Personas que fuman en la actualidad y la frecuencia es en un intervalo mayor que diaria

$\S$ Personas que declararon que ya no fuman
} 


\section{Cuadro IV}

Prevalencias de fumadores, en adultos con 20 años o más, 2000-2006

\begin{tabular}{|c|c|c|c|c|c|c|c|c|}
\hline & \multicolumn{4}{|c|}{ ENSA 2000} & \multicolumn{3}{|c|}{ ENSANUT 2006} & \multirow[b]{2}{*}{$\begin{array}{c}\text { No } \\
\text { fumador }\end{array}$} \\
\hline & $\begin{array}{c}\text { Fumador } \\
\text { diario }\end{array}$ & ocasional & Ex fumador & $\begin{array}{c}\text { No } \\
\text { fumador }\end{array}$ & $\begin{array}{c}\text { Fumador } \\
\text { diario }\end{array}$ & ocasional & Ex fumador & \\
\hline & $\begin{array}{l}\text { Porcentaje } \\
\text { (IC95\%) }\end{array}$ & $\begin{array}{l}\text { Porcentaje } \\
\text { (IC95\%) }\end{array}$ & $\begin{array}{l}\text { Porcentaje } \\
\text { (IC95\%) }\end{array}$ & $\begin{array}{l}\text { Porcentaje } \\
\text { (IC95\%) }\end{array}$ & $\begin{array}{l}\text { Porcentaje } \\
\text { (IC95\%) }\end{array}$ & $\begin{array}{l}\text { Porcentaje } \\
\text { (IC95\%) }\end{array}$ & $\begin{array}{l}\text { Porcentaje } \\
\text { (IC95\%) }\end{array}$ & $\begin{array}{l}\text { Porcentaje } \\
\text { (IC95\%) }\end{array}$ \\
\hline \multicolumn{9}{|l|}{ Grupos de edad } \\
\hline 20 a 29 años & $\begin{array}{c}11.6 \\
(10.2 \mathrm{a} \mid 3.1)\end{array}$ & $\begin{array}{c}11.5 \\
(10.4 \text { a I2.8) }\end{array}$ & $\begin{array}{c}8.6 \\
(7.8 \text { a } 9.4)\end{array}$ & $\begin{array}{c}68.3 \\
(66.6 \text { a } 70.0)\end{array}$ & $\begin{array}{c}13.8 \\
(12.6 \mathrm{a} \text { I5.I) }\end{array}$ & $\begin{array}{c}8.0 \\
(7.1 \text { a } 8.9)\end{array}$ & $\begin{array}{c}7.1 \\
(6.3 \text { a } 8.0)\end{array}$ & $\begin{array}{c}71.2 \\
(69.5 \text { a } 72.7)\end{array}$ \\
\hline 30 a 39 años & $\begin{array}{c}13.6 \\
(11.9 \text { a I5.4) }\end{array}$ & $\begin{array}{c}10.3 \\
(9.4 \text { a II.3) }\end{array}$ & $\begin{array}{c}12.5 \\
(11.5 \mathrm{a} \mathrm{I3.5})\end{array}$ & $\begin{array}{c}63.7 \\
\text { (61.8 a 65.5) }\end{array}$ & $\begin{array}{c}13.4 \\
(|2.5 \mathrm{a}| 4.3)\end{array}$ & $\begin{array}{c}6.5 \\
(5.8 \text { a } 7.4)\end{array}$ & $\begin{array}{c}8.0 \\
(7.2 \text { a } 8.8)\end{array}$ & $\begin{array}{c}72.1 \\
(70.8 \text { a } 73.3)\end{array}$ \\
\hline 40 a 49 años & $\begin{array}{c}\text { I6.I } \\
(14.5 \mathrm{a} \text { I7.9) }\end{array}$ & $\begin{array}{c}9.2 \\
(8.2 \mathrm{a} 10.4)\end{array}$ & $\begin{array}{c}15.2 \\
(14.0 \mathrm{a} \mathrm{I6.4})\end{array}$ & $\begin{array}{c}59.5 \\
(57.5 \mathrm{a} 61.4)\end{array}$ & $\begin{array}{c}14.7 \\
(13.5 \text { a I5.9) }\end{array}$ & $\begin{array}{c}5.3 \\
(4.6 \mathrm{a} 6.1)\end{array}$ & $\begin{array}{c}10.6 \\
(9.6 \mathrm{a} \mathrm{II.7)}\end{array}$ & $\begin{array}{c}69.4 \\
(67.8 \text { a } 70.9)\end{array}$ \\
\hline 50 a 59 años & $\begin{array}{c}14.2 \\
(\mid 2.5 \text { a I6.I })\end{array}$ & $\begin{array}{c}6.2 \\
(5.2 \text { a } 7.3)\end{array}$ & $\begin{array}{c}20.5 \\
(18.7 \text { a } 22.4)\end{array}$ & $\begin{array}{c}59.2 \\
(56.8 \text { a } 61.5)\end{array}$ & $\begin{array}{c}14.4 \\
(|3.0 \mathrm{a}| 6.0)\end{array}$ & $\begin{array}{c}4.6 \\
(3.9 \text { a } 5.5)\end{array}$ & $\begin{array}{c}14.6 \\
(\mid 3.1 \text { a I6.2) }\end{array}$ & $\begin{array}{c}66.3 \\
(64.4 \text { a } 68.3)\end{array}$ \\
\hline 60 a 69 años & $\begin{array}{c}11.9 \\
(10.3 \text { a I3.6) }\end{array}$ & $\begin{array}{c}4.6 \\
(3.8 \text { a } 5.6)\end{array}$ & $\begin{array}{c}22.2 \\
(20.1 \text { a } 24.4)\end{array}$ & $\begin{array}{c}61.3 \\
(58.7 \text { a } 63.8)\end{array}$ & $\begin{array}{c}11.9 \\
(10.5 \text { a I3.6) }\end{array}$ & $\begin{array}{c}2.4 \\
(1.7 \text { a } 3.4)\end{array}$ & $\begin{array}{c}\text { I5.I } \\
(\mid 3.3 \text { a I7.0) }\end{array}$ & $\begin{array}{c}70.6 \\
(68.3 \text { a } 72.8)\end{array}$ \\
\hline 70 a 79 años & $\begin{array}{c}7.9 \\
(6.3 \text { a } 9.8)\end{array}$ & $\begin{array}{c}4.0 \\
(2.8 \text { a } 5.5)\end{array}$ & $\begin{array}{c}26.8 \\
(23.6 \text { a } 30.2)\end{array}$ & $\begin{array}{c}61.4 \\
(27.4 \text { a } 65.2)\end{array}$ & $\begin{array}{c}8.9 \\
(7.4 \text { a I0.7) }\end{array}$ & $\begin{array}{c}1.7 \\
(1.2 \text { a } 2.5)\end{array}$ & $\begin{array}{c}19.5 \\
(17.3 \text { a 22.0) }\end{array}$ & $\begin{array}{c}69.8 \\
(67.0 \text { a } 72.5)\end{array}$ \\
\hline 80 años o más & $\begin{array}{c}7.2 \\
(4.8 \mathrm{a} \mid 0.5)\end{array}$ & $\begin{array}{c}3.0 \\
(1.8 \text { a } 5.0)\end{array}$ & $\begin{array}{c}24.4 \\
(20.2 \text { a } 29.2)\end{array}$ & $\begin{array}{c}65.4 \\
(60.5 \text { a } 70.0)\end{array}$ & $\begin{array}{c}5.0 \\
(3.6 \mathrm{a} 6.9)\end{array}$ & $\begin{array}{c}1.0 \\
(0.4 \text { a 2.5) }\end{array}$ & $\begin{array}{c}21.5 \\
(18.2 \text { a } 25.3)\end{array}$ & $\begin{array}{c}72.4 \\
(68.5 \text { a } 76 . \mathrm{I})\end{array}$ \\
\hline \multicolumn{9}{|l|}{ Tamaño de localidad } \\
\hline Menos de 2500 habitantes & $\begin{array}{c}7.6 \\
(6.6 \text { a 8.9) }\end{array}$ & $\begin{array}{c}8.1 \\
(7.2 \text { a } 9.2)\end{array}$ & $\begin{array}{c}12.3 \\
(11.1 \text { a I3.6) }\end{array}$ & $\begin{array}{c}72.0 \\
(69.8 \text { a } 74.0)\end{array}$ & $\begin{array}{c}8.1 \\
(7.3 \text { a } 8.9)\end{array}$ & $\begin{array}{c}4.2 \\
(3.5 \text { a } 5.1)\end{array}$ & $\begin{array}{c}7.9 \\
(7.1 \text { a } 8.7)\end{array}$ & $\begin{array}{c}79.8 \\
(78.6 \mathrm{a} 81.0)\end{array}$ \\
\hline 2500 habitantes o más & $\begin{array}{c}14.5 \\
(13.2 \mathrm{a} \text { |5.8) }\end{array}$ & $\begin{array}{c}9.8 \\
(9.2 \text { a } 10.4) \\
\end{array}$ & $\begin{array}{c}14.3 \\
(13.5 \text { a I5.I) }\end{array}$ & $\begin{array}{c}61.5 \\
(60.1 \text { a } 62.8)\end{array}$ & $\begin{array}{c}14.7 \\
(\mid 4.0 \text { a I5.3) }\end{array}$ & $\begin{array}{c}6.0 \\
(5.6 \text { a 6.4) }\end{array}$ & $\begin{array}{c}11.5 \\
(10.9 \text { a I2.I) }\end{array}$ & $\begin{array}{c}67.8 \\
(66.9 \text { a 68.7) }\end{array}$ \\
\hline \multicolumn{9}{|l|}{ Consumo de alcohol } \\
\hline Sí & $\begin{array}{c}20.3 \\
(18.7 \mathrm{a} 22.1)\end{array}$ & $\begin{array}{c}17.4 \\
(16.4 \mathrm{a} \text { I8.5) }\end{array}$ & $\begin{array}{c}16.1 \\
(15.1 \text { a I7.I) }\end{array}$ & $\begin{array}{c}46 . I \\
(44.6 \text { a } 47.6)\end{array}$ & $\begin{array}{c}23.2 \\
\text { (22.I a } 24.4)\end{array}$ & $\begin{array}{c}12.0 \\
(I 1 . \mid \text { a I2.9) }\end{array}$ & $\begin{array}{c}15.2 \\
(14.3 \text { a I6.2) }\end{array}$ & $\begin{array}{c}49.6 \\
(48.2 \text { a } 50.9)\end{array}$ \\
\hline No & $\begin{array}{c}14.3 \\
(12.7 \mathrm{a} \mid 6.2)\end{array}$ & $\begin{array}{c}6.2 \\
(5.4 \text { a } 7.1)\end{array}$ & $\begin{array}{c}26.5 \\
(24.828 .2)\end{array}$ & $\begin{array}{c}53.0 \\
(50.9 \text { a } 55.1)\end{array}$ & $\begin{array}{c}8.2 \\
(7.7 \text { a } 8.7)\end{array}$ & $\begin{array}{c}2.3 \\
\text { (2.I a } 2.7)\end{array}$ & $\begin{array}{c}8.4 \\
(7.9 \mathrm{a} 9.0)\end{array}$ & $\begin{array}{c}81.0 \\
(80.3 \mathrm{a} 81.8)\end{array}$ \\
\hline Total & $\begin{array}{c}12.9 \\
(|1.8 \mathrm{a}| 4.1)\end{array}$ & $\begin{array}{c}9.4 \\
(8.9 \text { a } 9.9)\end{array}$ & $\begin{array}{c}13.8 \\
(|3.2 \mathrm{a}| 4.5)\end{array}$ & $\begin{array}{c}63.9 \\
(62.6 \text { a } 65.2)\end{array}$ & $\begin{array}{c}13.3 \\
(12.8 \mathrm{a} \mid 3.8)\end{array}$ & $\begin{array}{c}5.6 \\
(5.3 \text { a 6.0) }\end{array}$ & $\begin{array}{c}10.8 \\
(10.2 \mathrm{a} \mid \mathrm{II.3})\end{array}$ & $\begin{array}{c}70.3 \\
(69.5 \text { a } 71.0)\end{array}$ \\
\hline$\overline{N^{*}}$ & 6418345 & 4647078 & 6864364 & 31748858 & 7972889 & 3378002 & 6446260 & 42120497 \\
\hline
\end{tabular}

tivas en ambas encuestas para la población adolescente, dado que se encontró que a medida que la edad aumenta las prevalencias también lo hacen. Sin embargo, en la población con 20 años o más, el comportamiento de los fumadores es diferente, y no se encontraron diferencias significativas entre ambas encuestas analizadas en relación con las prevalencias de aquellos que fuman a diario. No resultó igual entre los fumadores ocasionales, entre quienes se encontraron disminuciones significativas tanto en hombres como en mujeres al pasar de $15.3 \%$ (IC95\%: 14.3 a 16.4) a 8.8\% (IC95\%: 8.1 a 9.5) en hombres y de $4.0 \%$ (IC95\%: 3.6 a 4.5$)$ a $3.0 \%$ (IC95\%: 2.6 a 3.5$)$ en mujeres. Además, se encontraron diferencias de las prevalencias de fumadores ocasionales entre los grupos de edad, tipo de localidad y consumo de alcohol. Por su parte, la población que se consideró ex fumadora presentó diferencias significativas en hombres, al pasar de $21.6 \%$ (IC95\%: 20.5 a 22.7) en el año 2000 a 16.5\% (IC95\% :
15.6 a 17.4) en 2006. Además, entre los ex fumadores las prevalencias resultaron significativamente diferentes según la edad, con las mayores prevalencias en el grupo de 70 a 79 años, en donde fue de 26.8\%(IC95\%: 23.6 a 30.2) en 2000 y $19.5 \%$ (IC95\%: 17.3 a 22.0) en 2006.

\section{Discusión}

Las cifras publicadas con datos de la ENSA 2000 fueron: $21.5 \%$ de fumadores actuales, $13.3 \%$ de ex fumadores, $61.5 \%$ de no fumadores y $3.8 \%$ de no especificados. ${ }^{11}$ Los datos que se presentan en este estudio son de $22.3 \%$ de fumadores actuales (12.9\% fuma todos los d as y $9.4 \%$ lo realiza de manera ocasional), $13.8 \%$ de ex fumadores y $63.9 \%$ de los que no han fumado. Los incrementos en los porcentajes se deben a que en este estudio sólo se tomaron en cuenta las personas que contestaron las preguntas, es decir que se excluyeron los no especificados. 
Los resultados obtenidos en los adolescentes son consistentes con los hallazgos previos. La Encuesta de Evaluación de los Hogares Urbanos 2002, dirigida a familias que viven en pobreza extrema, exhibe que, a mayor edad, la probabilidad de fumar e ingerir alcohol se incrementa, sobre todo en hombres. ${ }^{12}$ Campuzano y colaboradores ${ }^{8}$ mencionan que para el grupo de adolescentes de 12 a 17 años encontraron que, a medida que la edad aumentaba, el antecedente de haber fumado alguna vez en la vida se elevaba de manera proporcional.

Aunque la definición de prevalencia de fumador actual, fumador diario y fumador ocasional es diferente a la considerada en otras encuestas, el presente estudio muestra resultados consistentes con lo encontrado en otras investigaciones. La aplicación más reciente de las Encuestas Nacionales de Adicciones se realizó en 2002 y revela que $23.5 \%$ de la población de 12 a 65 años fuma. ${ }^{9}$ En los resultados que se presentan en este art culo se menciona que para el año 2000 la prevalencia de fumador diario fue de $12.9 \%$ (IC95\%: 11.8 a 14.1) y la de fumador ocasional de $9.4 \%$ (IC95\%: 8.9 a 9.9); en 2006 las cifras disminuyeron a $13.3 \%($ IC95\%: 12.8 a 13.8) y $5.6 \%$ (IC95\%: 5.3 a 6.0), respectivamente. Es importante mencionar que estas cifras no admiten comparaciones directas, pues a pesar de que la ENA 2002 tiene representatividad de todo el pa s, las definiciones adoptadas de fumador actual no son iguales. En otro estudio ${ }^{13}$ se menciona que las cifras publicadas en la ENSA 2000 y en la ENA 2002 reflejan cierto grado de discrepancia; por ejemplo, para el grupo de 18 a 29 años, la ENA señala una prevalencia de fumadores actuales de $39.10 \%$ y la ENSA, para el grupo de 20 a 29 años, indica $22.0 \%$. El autor propone que la explicación para las diferencias observadas podr a radicar en las definiciones de fumador. Ambas fuentes consideran el hecho de haber fumado 100 cigarrillos, pero si bien la ENSA es expl cita en considerar el consumo en el momento de la encuesta, la ENA asume una definición más amplia al considerar el consumo en los últimos 12 meses.

Entre las limitaciones de este estudio se reconoce la de no contar con las preguntas adecuadas para considerar a los adolescentes como fumadores. Valdés Salgado y colaboradores ${ }^{14}$ establecen en la definición de prevalencia en el hábito de fumar que se considera como fumador a aquel joven que aceptó haber fumado al menos un cigarrillo en los últimos $30 \mathrm{~d}$ as (un mes) previos a la encuesta, y que esta definición está en concordancia con la definición adoptada por los centros para el Control y Prevención de Enfermedades de Estados Unidos (CDC, por sus siglas en inglés) en la Global Youth Tobacco Survey (GYTS) en 2003. Sin embargo, los datos que aqu se presentan aportan información importante acerca de los jóvenes que consumieron 100 cigarrillos o más durante su vida, y entre tales jóvenes se observa población menor de 12 años que ha consumido dicha cantidad de cigarrillos. Un aspecto importante es que la prevalencia en esta población joven disminuyó de 1.5 (IC 95\%: 1.0 a 2.3) a 0.4 (IC95\%: 0.2 a 0.8). No obstante, se requiere tener en cuenta que al utilizar la definición de 100 cigarrillos en la vida, en el grupo de adolescentes se subestima la prevalencia de fumadores, dado que por ser una población joven podr a estar iniciando la adicción y quedar excluida de la pregunta que realizan las fuentes de datos utilizadas en este trabajo.

Para finalizar, el presente estudio aporta información actualizada al 2006 acerca de las prevalencias en el consumo de tabaco. Sin embargo, es necesario que se continúen más estudios de manera minuciosa y que se incorpore la definición más consagrada en el mundo para que permita realizar comparaciones con otras encuestas realizadas en población mexicana y con encuestas internacionales.

\section{Referencias}

I. Secretaría de Salud. Plan Nacional de Salud 200I-2006. México: Secretaría de Salud, 2001.

2. Secretaría de Salud. Programa de Acción:Adicciones. Tabaquismo 200 I. México: Secretaría de Salud, 2001.

3. Kuri-Morales P, González-Roldán JF, Hoy MJ, Cortés-Ramírez M. Epidemiología del tabaquismo en México. Salud Publica Mex 2006;48 supl I:S9I-S98.

4.Valdés-Salgado R, Meneses-Gonzáles F, Lazcano-Ponce EC, HernándezRamos MI, Hernández-Ávila M. Encuesta sobre Tabaquismo en Jóvenes, México 2003. Cuernavaca: Instituto Nacional de Salud Pública, 2004.

5. Secretaría de Salud. Encuesta Nacional de adicciones 1998. México: Secretaría de Salud, 2002.

6. Kuri-Morales P, Revuelta-Herrera A, Tapia-Conyer R. Prevalencia del consumo del tabaco en México. Gac Med Mex 1995; 131:605-607.

7. Secretaría de Salud. Encuesta Nacional de Adicciones 1998 (Tabaco). México: Secretaría de Salud, 2000.

8. Campuzano-Rincón JC, Hernández-Ávila M, Samet JM, Méndez-Ramírez I,Tapia-Conyer R y Sepúlveda-Amor J. Comportamiento de los fumadores en México según las Encuestas Nacionales de Adicciones 1988 a 1998. En: Valdés-Salgado R, Lazcano-Ponce EC, Hernández-Ávila M. Primer informe sobre el combate al tabaquismo. México ante el Convenio Marco para el Control del Tabaco. Cuernavaca: Instituto Nacional de Salud Pública, 2005:21-27.

9. Instituto Nacional de Geografía e Informática (INEGI). Encuesta Nacional de Adicciones 2002. Aguascalientes: INEGI, 2004.

10. Palma O, Shama T, Franco A, Olaiz G y Méndez I. Metodología. En: Olaiz-Fernández G, Rivera-Dommarco J, Shamah-Levy T, Rojas R, Villalpando-Hernández S, Hernández Ávila M, Sepúlveda-Amor J. Encuesta Nacional de Salud y Nutrición 2006. Cuernavaca: Instituto Nacional de Salud Pública, 2006:19-23. 
II. Olaiz G, Rojas R, Barquera S, Shamah T,Aguilar C, Cravioto P, López P, Hernández M, Tapia R, Sepúlveda J. Encuesta Nacional de Salud 2000, Tomo 2. La salud de los adultos. Cuernavaca: Instituto Nacional de Salud Pública, 2003.

12. Urquieta JE, Hernández-Ávila M, Hernández B. El consumo de tabaco y alcohol en jóvenes de zonas urbanas marginadas de México. Un análisis de decisiones relacionadas. Salud Publica Mex 2006;48 supl I: S30-S40.

13.Valdés-Salgado R. Las cifras de la pandemia. Daños a la salud y mortalidad atribuible. En:Valdés-Salgado R, Lazcano-Ponce EC, Hernández-
Avila M. Primer informe sobre el combate al tabaquismo. México ante el Convenio Marco para el Control del Tabaco. Cuernavaca: Instituto Nacional de Salud Pública, 2005:29-4I.

14.Valdés-Salgado R, Thrasher J, Sánchez-Zamorano LM, Lazcano-Ponce E, Reynales-Shigematsu LM, Meneses-González F, Hernández-Avila M. Los retos del convenio marco para el control del tabaco en México: un diagnóstico a partir de la Encuesta sobre tabaquismo en jóvenes. Salud Publica Mex 2006;48:S5-SI6. 